

\title{
Coupled fluid-structure computational methods for aircraft ditching simulations: comparison of ALE-FE and SPH-FE approaches
}

\author{
M.H. Siemann, B. Langrand
}

\section{- To cite this version:}

M.H. Siemann, B. Langrand. Coupled fluid-structure computational methods for aircraft ditching simulations: comparison of ALE-FE and SPH-FE approaches. 2016. hal-01413180

\author{
HAL Id: hal-01413180 \\ https://hal.science/hal-01413180
}

Preprint submitted on 12 Dec 2016

HAL is a multi-disciplinary open access archive for the deposit and dissemination of scientific research documents, whether they are published or not. The documents may come from teaching and research institutions in France or abroad, or from public or private research centers.
L'archive ouverte pluridisciplinaire HAL, est destinée au dépôt et à la diffusion de documents scientifiques de niveau recherche, publiés ou non, émanant des établissements d'enseignement et de recherche français ou étrangers, des laboratoires publics ou privés. 


\title{
Coupled fluid-structure computational methods for aircraft ditching simulations: comparison of ALE-FE and SPH-FE approaches
}

\author{
M.H. Siemann ${ }^{\mathrm{a}, 1, *}$, B. Langrand ${ }^{\mathrm{b}, 2}$ \\ ${ }^{a}$ DLR - German Aerospace Center \\ ${ }^{b}$ Onera - The French Aerospace Lab
}

\section{Abstract}

This article deals with advanced, coupled numerical approaches for simulating the transient fluid structure-interaction as occurring during aircraft emergency landing on water, i.e. ditching. The structure is discretized using the Finite Element method, while for the fluid domain the Arbitrary LagrangianEulerian and the hybrid Smoothed Particle Hydrodynamics-Finite Element method are employed. First, the structural models are thoroughly validated ensuring differences arising from the use of two software packages to be as small as possible. Secondly, selected benchmark cases of guided ditching experiments are simulated with both numerical approaches and numerical results are compared with experimental data. A good agreement is found for pressure, normal force, and strain time histories. Finally, modeling aspects affecting the results such as the effects of the plasticity computation algorithm, air, and model simplifications are discussed.

Keywords: Aircraft ditching, Fluid-structure interaction, Arbitrary Lagrangian-Eulerian, Coupled Euler-Lagrangian, Smoothed Particle Hydrodynamics

\footnotetext{
${ }^{*}$ Corresponding author: Tel.: +49 7116862 297; fax: +49 7116862227 .

Email addresses: martin.siemann@dlr.de (M.H. Siemann), bertrand.langrand@onera.fr (B. Langrand)

${ }^{1}$ Institute of Structures and Design, 70569 Stuttgart, Germany

${ }^{2}$ Aeroelasticity and Structural Dynamics Department, F-59014 Lille Cedex, France
} 


\section{Introduction}

The majority of the air transport occurs over significant bodies of water and thus the emergency landing on water (ditching) represents a fortunately rare but significant safety threat. In order to minimize the risk of immediate injuries to the occupants and to allow for a safe evacuation of the aircraft before it potentially sinks, aircraft manufacturers must demonstrate the structural capabilities within the certification process of novel aircraft types (see [1-3] for further details about certification regulations and processes). While ditching analyses are conducted for fixed-wing aircraft $[1,2,4,5]$ and helicopters $[3,6-8]$, the present work considers solely fixed-wing aircraft ditching. The extreme characteristics of ditching constitute great challenges for the numerical simulation. For instance, capturing the fluid dynamics, such as jet and bow wave formation and breakup, separation, and splashing, over typically long time scales is very challenging. Moreover, the involved hydrodynamic effects and the highly transient hydrodynamic pressure acting on the structure pose challenges for the numerical simulation. Furthermore, the nonlinear structural behavior with potential rupture and the complex fluid-structure interaction (FSI) occurring during a ditching constitute additional challenges for the simulation. Other major challenges arise from the multi-scale character of the phenomena involved in a ditching. On the one hand, acting pressures rise and fall within milliseconds requiring very small time steps to be resolved, yet the impact phase lasts several hundred milliseconds. On the other hand, the high impact pressures act over very local zones in the order of millimeters, while the aircraft structure moves several meters throughout the event. These scale differences, which amount to about a factor of one thousand in both time and space, account for major challenges for the computational models that need to resolve the local scales, while remaining computationally efficient to allow covering the complete impact phase.

Due to the challenges addressed above, the state-of-the-art analysis methods are currently limited to comparisons of the novel structural design with aircraft structures that were previously certified for ditching, sub-scale experiments, and (semi-)analytical methods in combination with subsequent, uncoupled finite element analyses of the structure. A detailed description as well as associated advantages and disadvantages are given in $[1,2]$. In summary, current means widely lack the ability to be applied to novel and uncommon designs thus limiting progress, they are based on several assumptions and simplifications that cause inaccuracies, and, perhaps more critical, 
they do not account for the coupled fluid-structure interaction. To the knowledge of the authors, the latter is a topic of research since the 1960s, where effects of structural deformations on hydrodynamic results were studied for purely vertical impact on water, e.g. [9, 10], and continues to be investigated nowadays, e.g. [11, 12]. Also for oblique impact on water at high horizontal velocity, structural deformations were demonstrated to significantly affect the acting hydrodynamic loads [1]. Thus, it appears to be essential to couple fluid and structure numerical solutions within advanced computational methods in order to correctly predict the structural response and to analyze the structural capacity of novel aircraft designs. Nevertheless, the large computational effort required currently limits the application of advanced, coupled numerical approaches to cases with simple structural models. Furthermore, comprehensive validation remains to be demonstrated as only few validation studies involving deformable structures impacting water at high horizontal velocity were conducted [1].

The term advanced, coupled numerical approaches comprises approaches that use (a) high-fidelity solutions for both the fluid and the structural domain and (b) a method to couple the fluid and the structural solution in the same computation. Typically, the explicit Finite Element (FE) method is adopted to simulate the structural response, as it is adapted to fast-transient dynamic problems, such as water impact, and it can portray complex 3D geometries and non-linear structural responses with many contacts, whereas fundamentally different computational methods are used to simulate the fluid behavior. Common mesh-based methods are pure Eulerian or Arbitrary Lagrangian-Eulerian (ALE) methods [13-15]. Common mesh-free methods comprise Smoothed Particle Hydrodynamics (SPH) [16] and Element-Free Galerkin (EFG) $[17,18]$ methods.

A general review on hull slamming, which is closely related to ditching, was given by Abrate [19]. The author provided an overview of the computational approaches used for water impact simulations comprising Boundary Element, SPH, FE, and fixed grid methods (Euler) including techniques to detect the interface between the domains (e.g. the water free surface) such as Cubic Interpolated Pseudo-particle, Volume of Fluid, and Level-Set method. For a test case of pure vertical water impact, a comparison of different methods to simulate the fluid has been carried out by Anghileri et al. [20]. The authors compared experimental and numerical simulation results of four different 
methods, i.e. FE, ALE, SPH, and EFG. They point out a dependence of the ALE results on the FSI coupling parameters and that finding a proper $\mathrm{SPH}$ discretization fineness is not trivial and thus time-consuming. The results of FEM simulations were found to give proper results only as long as fluid deformations are small and the EFG solution was computationally very expensive. Hughes et al. [21] presented an insight into recent work using FE, ALE, SPH, and EFG methods, yet with a focus on the application of the SPH method.

Guo et al. [22] employed the ALE method investigating the effect of the initial pitch angle on the aircraft motion during the ditching. The ALE method was also used by Qu et al. [23], who refer to their approach as global-moving-mesh method, to study the ditching characteristics of a generic aircraft fuselage rigid body model, NACA2929 A-body [24]. Pentecôte and Kohlgrüber [25] compared the capabilities of both FE and SPH method to simulate the ditching of a transport aircraft rigid body model. They concluded that the high horizontal velocity of the aircraft together with the considerable duration of the event leads to large mesh distortions that result in a severe drop of the critical time step and eventually to an early termination of the simulation. Another application of the SPH method to a ditching simulation was reported by Ortiz et al. [5], who simulated a deformable Airbus A321 model impacting water and reported the feasibility of the method yet with extremely large runtimes. Climent et al. [2] presented the ditching analysis of a military transport aircraft applying both the FE and the SPH method. Toso [3] employed the SPH method to simulate the water impact of a generic aircraft fuselage rigid body model, NACA2929 A-body [24]. Groenenboom and Siemann [26] simulated the water impact of generic panels of the guided ditching experimental campaign (cf. Sec. 3.1) using the SPH method. The authors furthermore presented modeling techniques that permit a significant efficiency increase.

In summary, the large mesh distortion due to the presence of a high horizontal velocity renders the use of Lagrangian mesh-based methods, such as the FE method, impractical if not impossible. Furthermore, to the knowledge of the authors there exists no application of the EFG method to water impact cases with horizontal velocity. Therefore, the two most used methods, ALE and SPH, are further investigated in this work.

The main objective of this work is to compare and to assess the capabilities as well as the limitations of the two most commonly used, yet fundamentally 
different, coupled numerical approaches, ALE-FE and SPH-FE, based on a recent benchmark experiment for ditching analysis involving both quasi-rigid and deformable structures. This work employs two explicit software packages, which provide a wide range of structural modeling capabilities that have been demonstrated to work within various applications.

The remainder of the article is organized as follows: Section 2 provides a brief description of the adopted numerical approaches, which serves as a basis for the later comparison and assessment. The selected benchmark experiment and the developed simulation models are presented in Section 3. Section 4 compares experimental and numerical results, followed by a discussion of the capabilities and limitation of the computational approaches investigated. Finally, conclusions are drawn and directions for future research are outlined.

\section{Numerical Approaches}

The Lagrangian formulation is classical in solid mechanics and structural analysis. The mesh and the material points are tied and the mesh and material deformations are consequently linked. Sliding between material (structure) and mesh is not allowed. Loads and boundary conditions can easily be applied to the material points (nodes). The Lagrangian description tracks easily the free surfaces and interfaces between different materials. However, when the structure is severely deformed, Lagrangian elements become similarly distorted since they follow the material deformation. Therefore, in those cases the accuracy and robustness of the Lagrangian simulations decrease severely.

The Eulerian formulation is classical in fluid mechanics. The mesh is fixed and the material flows through the mesh. Equations are modified with respect to Lagrangian formulation in order to take into account the convective terms. The treatment of moving boundaries and interfaces is difficult with Eulerian elements. The Eulerian formulation cannot be used in many cases where the boundaries of the domain move.

In the Arbitrary Lagrangian-Eulerian (ALE) formulation, the material flows through an arbitrary moving mesh. Both the material and the mesh move with respect to the laboratory. It looks like a combination of Lagrangian and Eulerian formulations. Grid velocities and displacements are arbitrary. In practice, built-in algorithms determine smooth grid deformation according to displacements of the ALE domain boundaries. Several 
algorithms are available, e.g. Donea et al. [27]. Note that the ALE formulation can be degenerated in Lagrangian (the grid velocity is equal to the material velocity) or in Eulerian (the grid velocity is set to zero).

Common between the regarded approaches, the structure is discretized using the Finite Element method. For the fluid domain, however, there are several methods available. This section describes the fundamentals of fluid and structural modeling as well as the fluid-structure interaction. The formulations presented in this section use the explicit approach for obtaining numerical approximations to the solutions of time-dependent ordinary and partial differential equations.

\subsection{Fundamentals of Fluid Modeling}

The fundamental system of differential equations to be solved within the fluid domain comprises the so-called Euler equations:

$$
\begin{aligned}
\text { conservation of mass } & \frac{\mathrm{d} \rho}{\mathrm{d} t}=-\rho \nabla \cdot \mathbf{v}, \\
\text { conservation of momentum } & \frac{\mathrm{d} \mathbf{v}}{\mathrm{d} t}=-\frac{1}{\rho} \nabla p+\mathbf{g}, \\
\text { conservation of energy } & \frac{\mathrm{d} u}{\mathrm{~d} t}=-\frac{p}{\rho} \nabla \cdot \mathbf{v} .
\end{aligned}
$$

Above symbols refer to density $\rho$, time $t$, velocity vector $\mathbf{v}$, pressure $p$, vector of accelerations resulting from external body forces $\mathbf{g}$ (i.e. gravitation acceleration), and specific internal energy $u$. The Euler equations neglect effects of fluid viscosity, surface tension, and temperature. These effects are of minor importance for water impact at high velocity, which is dominated by inertial and pressure forces [3, 19, 28].

The fundamentals of the adopted computational methods to simulate the fluid are not repeated here; the reader can refer to $[13,27]$ and to $[29,30]$ for the ALE and the SPH formulations respectively. Both formulations are based on the Euler equations for the fluid modeling.

\subsection{Fundamentals of Structural Modeling}

Typical modeling techniques used within the field of aeronautical engineering crash simulations are adopted. The thin walled structure of the aircraft is discretized using thin bi-linear shell elements. In order to portray nonlinear structural deformations, elastic-(visco)plastic material models in 
conjunction with an appropriately refined discretization able to capture the transient response of the structure are commonly employed. The failure of the material can be modeled in different ways, from the basic element erosion technique to the advanced extended FE method.

\subsection{Modeling Fluid-Structure Interaction}

There are several possibilities to couple the fluid and the structural solution such as coupling based on constraint equations, where fluid and structural nodes must obey kinematic constraints, or penalty contact formulations, where a small amount of penetration is permitted and subsequently a repulsive force is generated.

\subsubsection{Coupled Euler-Lagrange Method}

Among the different methods described by Casadei and co-workers to couple fluids and a structure [31-33], the coupling between fluids and structure relies, in the computations presented in the paper, on an immersed interface also referred to as embedded interface. The structure and the fluids are meshed in a completely different manner and the structure mesh is immersed within the mesh of the fluids [33]. An unstructured fluid mesh (conforming or non-conforming) is no longer necessary. With this technique, the fluid mesh can be even regular. In this case, an ALE formulation is no longer necessary in the fluid sub-domains that can be Eulerian so than the fluid mesh will never entangle (fixed mesh). The immersed interface allows slave nodes (material grid) belonging to the Euler or ALE fluid media to interact with the master surfaces of the Lagrange solid medium. The coupling forces are computed using the penalty method that depends on two parameters: the contact height $h_{C}$ and the contact stiffness $k_{C}$ that are defined by Eq. (4) and (5) respectively. This interface was applied to deal with vertical water impact problems such as presented in [34] for rotor-craft.

$$
\begin{aligned}
h_{C} & \geq 1.5 \cdot l_{F} \\
k_{C} & =\frac{\rho_{F} v^{2}}{h_{C}} A_{S}
\end{aligned}
$$

where $l_{F}$ is the fluid cell size (Euler or ALE), $\rho_{F}$ the fluid density, $v$ is the relative velocity between the fluid and the structure in the impact direction (here the norm of the horizontal and vertical velocity components), and $A_{S}$ 
is the area of the structural elements. The factor of 1.5 is needed to maintain contact once it is established. If the mesh size of the fluid and the structure are both homogeneous in the interaction area, the fluid structure coupling can be easily defined with one interface only.

When the fluid penetrates the contact area, a contact force is applied to the degrees of freedom (DOF) of the fluid material as well as to the DOF of the fluid grid. This contact force is proportional to the penetration depth.

\subsubsection{Coupled SPH-FE Method}

A weak coupling using a node-to-surface penalty contact algorithm is typically adopted. Therein, the particles represent the slave nodes and the structural elements are the master segments. The contact algorithm checks for any penetration of slave nodes into the contact zone around the master segments, which is defined by an offset commonly referred to as contact height $h_{C}$. This contact height is chosen in relation to the thickness of the structure $t_{S}$ and the particle spacing $d_{F}$ as

$$
h_{C}=0.5\left(t_{S}+d_{F}\right) .
$$

Upon penetration, a repulsive force is applied to the particle and, in order to conserve momentum, the force magnitude is distributed to the element's nodes in opposed direction. The contact force magnitude is proportional to the penetration depth and to the contact stiffness. Additionally, a velocityproportional damping is included to dissipate numerical oscillations.

\section{Benchmark Simulations}

\subsection{Benchmark Experiment}

The present study uses selected benchmark test cases from the guided ditching experimental campaign, which was conducted by CNR-INSEAN ${ }^{3}$ as part of the EC-funded research project SMAES ${ }^{4}$. A detailed description of the guided ditching facility, the experimental campaign, as well as analyzes of uncertainties and results can be found in $[1,35,36]$. Subsequently, a brief insight is given.

\footnotetext{
${ }^{3}$ Consiglio Nazionale delle Ricerche - Istituto Nazionale per Studi ed Esperienze di Architettura Navale

${ }^{4}$ SMart Aircraft in Emergency Situations, 2011-2014
} 
The guided ditching experimental facility comprises a large water tank and a guide structure, which features five height-adjustable bridges holding the guide track. A trolley carrying the test specimens is attached to the guide track, which permits only a motion in the direction of the guide track. In addition, there is a catapult-like acceleration system that accelerates the specimens to the high impact velocities characteristic for fixed-wing aircraft ditching at quasi-full-scale. The design of the facility permits to vary the guide track inclination, i.e. the ratio of vertical to horizontal impact velocity, in a range representative of realistic full-scale aircraft ditching conditions. All specimens analyzed within this experimental campaign measured $1000 \mathrm{~mm}$ in length and $500 \mathrm{~mm}$ in width, yet their thickness, material, and transverse curvature were varied. In total, 22 test cases with typically two to three repeats were investigated. Two selected test cases were repeated ten times in order to demonstrate the repeatability of the experiments, which proved to be high [36]. The experimental campaign produced a comprehensive set of highly repetitive, high-quality data, which are used for the purpose of comparison and assessment of the numerical methods within the present work.

For the present work, three test cases with flat specimens of different thickness $(15 \mathrm{~mm}, 3 \mathrm{~mm}$, and $0.8 \mathrm{~mm}$ noted as case 1122, 2122, and 3122, respectively) made of aluminum alloy Al2024 were selected. The three specimens were tested with identical impact conditions of $6^{\circ}$ pitch angle, $40 \mathrm{~m} / \mathrm{s}$ initial horizontal, and $-1.5 \mathrm{~m} / \mathrm{s}$ initial vertical impact velocity. The specimens were attached to quasi-rigid, L-shaped frames, reducing the unsupported area to $850 \times 350 \mathrm{~mm}$ (case 1122) and $900 \times 400 \mathrm{~mm}$ (cases 2122 and 3122 ). The total mass undergoing the impact was $840 \mathrm{~kg}, 834 \mathrm{~kg}$, and $832 \mathrm{~kg}$ for the case 1122,2122 , and 3122 , respectively.

The complete instrumentation was presented in $[35,36]$. Here results from load cells measuring forces acting in normal direction of the panel, biaxial strain gauges, and pressure probes are used. Experimental results were sampled at $20 \mathrm{kHz}$ except pressure results that were sampled at $200 \mathrm{kHz}$ due to the highly transient nature of the hydrodynamic pressure.

\subsection{Simulation Model}

In this section, the fluid and structural models employed are described along with an overview of the hardware and the computational settings adopted for the simulations in Tab. 1. 
Table 1: Overview of hardware and computational settings used for ALE-FE and SPH-FE computations.

\begin{tabular}{lll}
\hline & ALE-FE simulations & \multicolumn{1}{c}{ SPH-FE simulations } \\
\hline Cluster system & Linux CentOS 6.5 & Linux Ubuntu, Beowulf \\
Processor type & $2 \times$ Intel Ivy-Bridge & $2 \times$ Intel Xeon E5540 \\
& E5-2667v2 (8-core, & $(4-$ core, 64-bit, $2.53 \mathrm{GHz})$ \\
& $64-$ bit, 3.3 GHz) & \\
Number of CPUs & 32 & 8 \\
RAM per node & $16 \mathrm{~GB}$ & $24 \mathrm{~GB}$ \\
Computing mode & Distributed memory, & Shared memory, \\
& single precision & single precision \\
\hline
\end{tabular}

\subsubsection{Fluid Model: ALE}

The material behavior of the fluids is modeled using the hydrodynamic viscous fluid law given in Eq. (7) and (8)), which has been specifically designed to model liquids and gases.

$$
\begin{gathered}
s_{i j}=2 \rho \nu \dot{e}_{i j} \\
p=C_{0}+C_{1} \mu+C_{2} \mu^{2}+C_{3} \mu^{3}+\left(C_{4}+C_{5} \mu\right) E_{n} \text { with } \mu=\rho / \rho_{0}-1
\end{gathered}
$$

where $C_{i}$ and $E_{n}$ are material constants, $s_{i j}$ is the deviatoric tensor, $\nu$ the kinematic viscosity, and $\dot{e}_{i j}$ the deviatoric strain rate tensor.

For a quasi-incompressible medium like water, Eq. (8) is simplified to

$$
p=C_{0}+C_{1} \mu
$$

where $C_{0}$ is the hydrostatic pressure computed for the given depth and $C_{1}=\rho_{0} c^{2}=2.25 \mathrm{GPa}$ (with density $\rho_{0}=1 \cdot 10^{-6} \mathrm{~kg} / \mathrm{mm}^{3}$ and speed of sound $c=1500 \mathrm{~m} / \mathrm{s})$. In addition, a minimum pressure $\left(p_{\min }\right)$ can be introduced in the hydrodynamic viscous fluid law to take cavitation effects into account. For water, this minimum pressure is set to $1 \cdot 10^{-23} \mathrm{GPa}$.

The air is assumed to behave like an ideal gas. Thus, for air Eq. (8) is simplified to

$$
p=\left(C_{4}+C_{5} \mu\right) E_{n} .
$$

The material constants are totally defined as functions of the perfect gas constant $\gamma$ and the hydrostatic pressure $p_{0}$ as $C_{4}=C_{5}=\gamma-1$ and $E_{n}=p_{0} /(\gamma-1)$. 
With values of $\gamma=1.4$ and $p_{0}=0.1 \cdot 10^{-3} \mathrm{GPa}$ for the air, $C_{4}=C_{5}=0.4$ and $E_{n}=0.25 \cdot 10^{-3} \mathrm{~J}$. Furthermore, the air density is set to $\rho_{0}=1.2 \cdot 10^{-9} \mathrm{~kg} / \mathrm{mm}^{3}$.

In order to initialize the fluid domain with the hydrostatic pressure $\left(p_{0}=\right.$ $\rho_{0} g h$, with $h$ being the depth of water and $g$ the gravitational acceleration), the water domain is split in the vertical direction into layers and a specific material model is applied to each layer with the constant $C_{0}$ in Eq. (9) set to the depth-dependent hydrostatic pressure $p_{0}(h)$. The hydrostatic pressure field is balanced by the gravitational acceleration at the step zero of the computation and does not require preliminary computational steps.

To move the fluid domain with the translational velocity of the structure, ALE links are used between the nodes of the fluid domain (slaves) and one node of the structure (master), for which the center of gravity node of the given structure is selected. With the translational velocity of the master and slave nodes being assigned to the same value, the computation only requires the fluid volume surrounding the structure since the fluid domain (grid) translates with the global displacement of the structure. On the one hand, it is possible to reduce considerably the size of the fluid domain, which is very interesting for ditching applications, and to adapt the fluid mesh in the FSI zone to improve the computation accuracy. Consequently the number of fluid elements and the computational cost are reduced. On the other hand, it is necessary to implement inlets and outlets in the computation to inject and eject fluid material when the fluid domain translates with the structure. Inlet and outlet are used as boundary conditions of the fluid domain. Generally the following elementary boundary conditions are used:

- For inlet, flux is imposed using imposed velocities (density and energy are imposed as constants). Continuity is imposed for pressure (display purposes only).

- For outlet, continuity is imposed for all variables except pressure. When using the silent boundary option, a value for sound speed and a typical relaxation length are provided, which must be greater than the largest wave length of interest.

To avoid any interaction between the inlets/outlets and the impact zone, it is advised to insert some fluid elements between the inlets/outlets and the impact zone: ahead the structure, more than the length of the structure, and behind the structure, more than twice the length of the structure. 

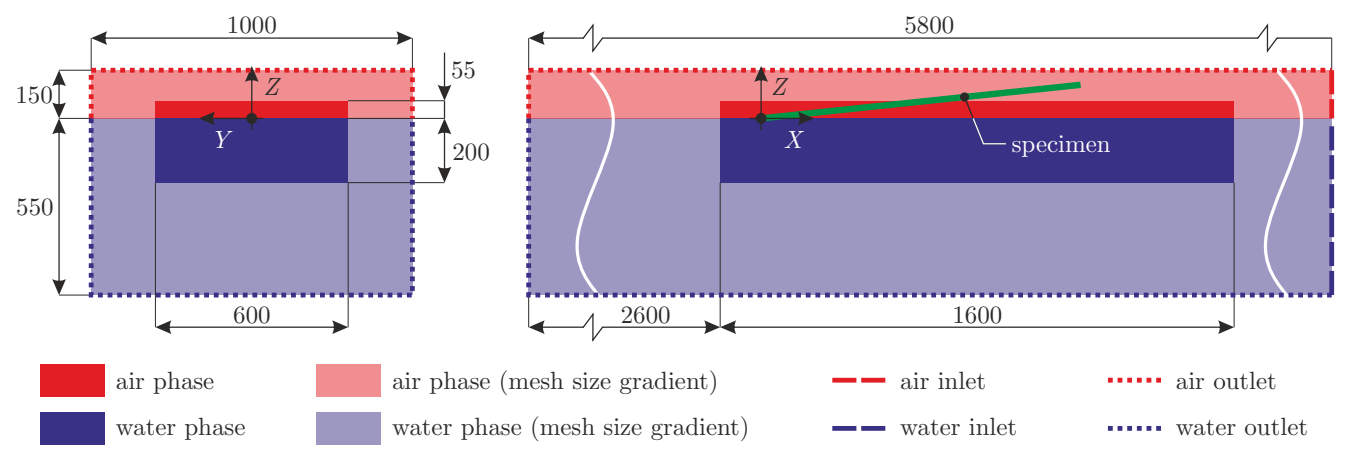

Figure 1: Illustration of ALE fluid domain. All measures are in millimeters.

The fluid domain (air and water) is modeled using 3D hexahedron finite elements ( 8 nodes) and the ALE formulation is considered. Figure 1 shows the fluid model and provides its dimensions. The mesh size in the impact zone is about $5 \mathrm{~mm}$ (lower than the mesh size of the structure, in order to load the shell FE with more than a single fluid hexahedron FE). The length of the structure being about $1000 \mathrm{~mm}$, the distance between the impact zone and the inlets is $1600 \mathrm{~mm}$ (more than $1000 \mathrm{~mm}$ ) and the distance between the outlets and the impact zone is $2600 \mathrm{~mm}$ (more than $2000 \mathrm{~mm}$ ). In order to reduce the total number of elements while keeping a good resolution in the impact zone, the mesh size is increased in all directions with a growth rate of about $10 \%$ outside the impact zone. The total length of the fluid domain is $5800 \mathrm{~mm}$ leading to a total volume of $4.06 \cdot 10^{9} \mathrm{~mm}^{3}$. The total number elements is about 1.27 million and 3.68 million for air and water respectively. Figure 1 displays the ALE fluid model. The fluids are initially at rest and gravity is applied.

\subsubsection{Fluid Model: SPH-FE}

For the SPH simulations, the Tait equation of state (11), which models a weakly-compressible fluid, is adopted. It reads

$$
p(\rho)=p_{0}+\frac{\rho_{0} c^{2}}{\gamma}\left[\left(\frac{\rho}{\rho_{0}}\right)^{\gamma}-1\right]
$$

with hydrostatic pressure $p_{0}=0$, density $\rho_{0}=1 \cdot 10^{-6} \mathrm{~kg} / \mathrm{mm}^{3}$, speed of sound $c_{0}=500 \mathrm{~m} / \mathrm{s}$, and adiabatic exponent $\gamma=7$ for water. Reducing the speed of sound, thus increasing the compressibility, is common practice for SPH simulations where bulk flow velocities remain well below the artificial speed 
of sound, e.g. below $10 \%$ [30]. It effectively reduces the strength of numerical pressure fluctuations. Computed pressure values below a defined minimum pressure are cut-off accounting for the effect of cavitation.

In contrast to the ALE fluid model, air is not accounted for in the SPHFE simulations due to the large number of particles additionally required. Nevertheless, the modeling of air and water in one simulation is technically possible. It requires, however, adapting the SPH scheme, i.e. by using another formulation for the pressure term in the momentum and energy equations that copes with the large density gradient of approximately $1.2 / 1000$ at the air-water interface ${ }^{5}$.

The SPH-FE fluid model adopts a hybrid approach combining the SPH method in the impact zone with the FE method (3D hexahedron finite elements, 8 nodes) in the surrounding far field. Selecting this modeling approach is motivated by the significantly higher computational cost of the SPH method in comparison to that of the FE method for an equivalent reference volume. Therefore, this hybrid modeling approach permits increasing the efficiency of the simulation model by far. Moreover, it was proven to work stable and robust within simulations of vertical water impact of simple, generic structures, helicopters, and rigid body fixed-wing aircraft [2, 3, 25, 37].

In order to further increase the efficiency of the computation, the translating active domain feature is employed. It defines a rectangular domain that moves with the impacting structure in reference to a selected node of the structure, which in the present cases is the trolley's rigid body reference node. Particles located within the active domain are considered within the SPH algorithm, whereas particles located outside of it are skipped. This increases the efficiency of the simulation by a factor approximately proportional to the ratio of the total number of particles to the number of active particles. For the present fluid model, this factor is approximately equal to 2.75 .

Figure 2 shows the fluid model and provides its dimensions, which are constant among all test cases. Particles are initially arranged in a hexagonal pattern with a spacing of $10 \mathrm{~mm}$. This particle spacing is defined in accordance with the shell element size of the structure that should be larger than the particle spacing to avoid an unphysical loading of the structure. The surrounding FE mesh size is about $30 \mathrm{~mm}$. The cross-section of the SPH-FE

\footnotetext{
${ }^{5}$ Further information is given in [1].
} 


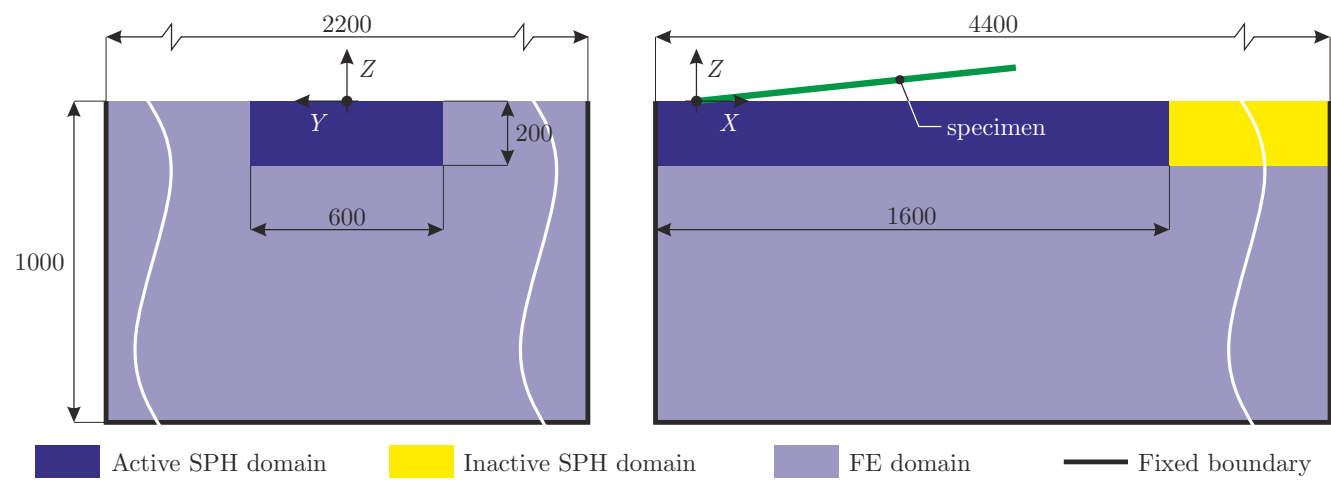

Figure 2: Illustration of hybrid SPH-FE fluid domain with translating active domain feature. All measures are in millimeters.

fluid domain is larger than that of the ALE fluid domain because there are no outlets; therefore, the fluid domain must be larger to avoid any influence due to the fixed boundaries (e.g. pressure wave reflections). Nevertheless, the size of the SPH domain equals that of the ALE water domain with constant mesh size $(1600 \mathrm{~mm} \times 600 \mathrm{~mm} \times 200 \mathrm{~mm})$. The total length of the fluid domain is $4400 \mathrm{~mm}$ resulting in a total volume of $9.68 \cdot 10^{9} \mathrm{~mm}^{3}$. In total there are about 1.06 million particles and 358000 volume elements composing the fluid domain. The fluid is initially at rest and gravity is applied.

\subsubsection{Structural Model}

The structural model contains the trolley box structure, the L-frame, and the panel as shown in Fig. 3. The trolley box structure and the L-frame form one rigid body. All structural parts are modeled using thin shell four-node bi-linear elements with a full-integration rule in the element plane and five integration points through the thickness. The characteristic element size on the panel is $10 \times 10 \mathrm{~mm}$. The panel is connected to the L-frame by means of point link elements with realistic stiffness in normal and shear directions calculated using the Huth-formula [38].

In order to reduce the code-specific differences between the simulation models to a minimum, the structural model was simplified. On the one hand, the guide structure is not modeled although small deformations were measured during the experiment [36]. On the other hand, the trolley model only contains the box structure and the L-frame, whereas the upper part of the trolley is not modeled. Furthermore, the box structure and the L- 


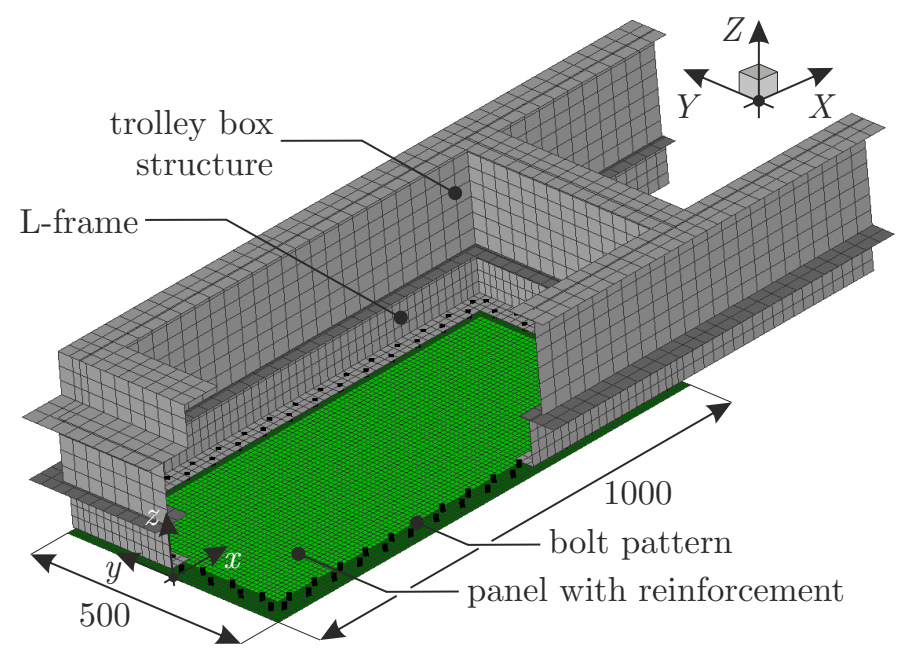

Figure 3: Illustration of structural model. Partially elements are hidden for clarity of illustration. All measures are in millimeters.

frame are defined to be rigid. Effects of these simplifications are discussed in Sec. 5.3.

The material behavior of the specimens uses an isotropic, elastic-plastic model with the isotropic hardening defined with the Ramberg-Osgood equation: $\sigma_{y}=\sigma_{0}+K p^{n}$. The elastic modulus $E=71 \mathrm{GPa}$, Poisson ratio $\nu=$ 0.32 , initial yield stress $\sigma_{0}=0.328 \mathrm{GPa}$, hardening modulus $K=0.768 \mathrm{GPa}$, and hardening exponent $n=0.5805$ were obtained from experiments performed by Onera on sheet metal plates of Al2024-T351. A Newton iterative algorithm available in both software packages is applied to compute the plastic variables. The density was set to $\rho=2.8 \cdot 10^{-6} \mathrm{~kg} / \mathrm{m}^{3}$ in the computations.

Boundary conditions are prescribed to the rigid body reference node of the structural model. The structure is free to move only along the path defined by the resulting vector of horizontal and vertical impact velocities, whereas all other degrees of freedom are constrained. In addition, the initial velocity used in the experiments is prescribed. All parts of the structure are subject to gravity. At the beginning of the impact, the structure is positioned with the specimen trailing edge at a distance of $X=100 \mathrm{~mm}$ from the rear of the ALE fluid domain with constant mesh size or the SPH active domain, respectively.

The total mass of the impacting structure is adjusted to match the experiments, e.g. $840 \mathrm{~kg}, 834 \mathrm{~kg}$, and $832 \mathrm{~kg}$ for test cases 1122, 2122, and 3122, 


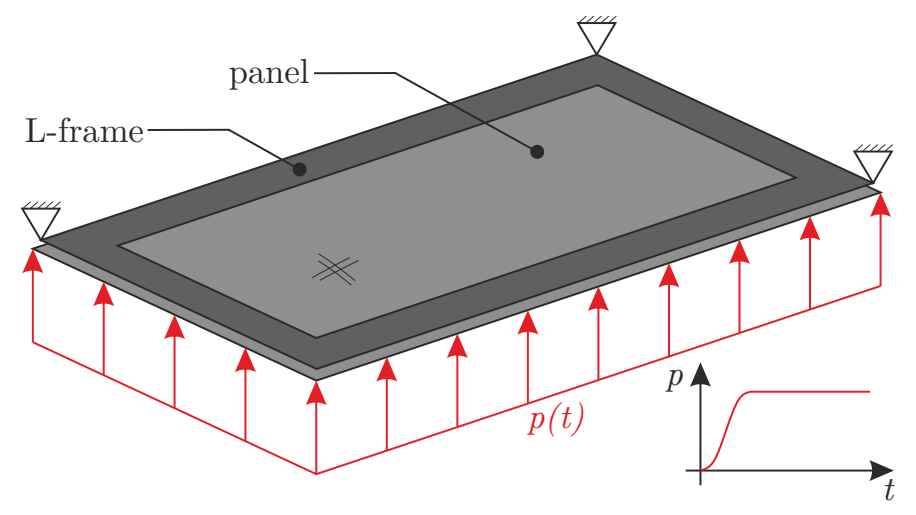

Figure 4: Validation test case for structural models. The horizontal portion of the L-frame is fixed in all degrees of freedom.

respectively. This is done by adding a concentrated mass to the rigid body reference node that accounts for parts not modeled such as the acquisition system.

\subsection{Validation of Structural Model}

In order to validate the structural model adopted within each software package, its response to a predefined pressure load is compared using the test case shown in Fig. 4. The structural model contains solely the lower portion of the rigid L-frame and the panel. For the present cases, the Lframe is fixed in space and the panel is loaded with a predefined pressuretime history. The applied pressure loading on the panel is composed of a sinusoidal ramp, $f_{p}$, over $5 \mathrm{~ms}$ up to a constant pressure of $1 \cdot 10^{-3} \mathrm{GPa}$, which is arbitrary, yet in the order of the peak pressures measured in the experiments, until the end of the simulation at $20 \mathrm{~ms}$. The sinusoidal ramp is defined as $f_{p}=0.5 \times(1+\sin ((1+t) \pi))$ and scaled accordingly.

For the purpose of validation, maximum deformations normal to the panel surface and in transverse direction as well as strain distributions and time histories are compared. Table 2 provides the maximum deformations and their relative differences, which are up to about $3 \%, 17 \%$, and $7 \%$ for cases 1122,2122 , and 3122 , respectively.

Figure 5 shows the upper surface strain distributions in $x$-direction $\left(\varepsilon_{x x}\right)$ for the test cases 2122 and 3122 . The strain distributions obtained by the different software packages for the same test cases show a good agreement. Small differences can however be observed near the boundaries, where joints 
Table 2: Maximum deformations for structural model validation test case at $t=20 \mathrm{~ms}$.

\begin{tabular}{rrrrrrr}
\hline & \multicolumn{4}{c}{$\Delta Y_{\max }$} & \multicolumn{3}{c}{$\Delta Z_{\max }$} \\
\cline { 2 - 7 } Case & ALE & SPH & Difference [\%] & ALE & SPH-FE & Difference [\%] \\
\hline 1122 & 0.021 & 0.022 & 2.09 & 2.46 & 2.61 & 2.90 \\
2122 & 3.590 & 5.030 & 16.71 & 33.60 & 41.66 & 10.71 \\
3122 & 2.890 & 3.350 & 7.37 & 56.62 & 54.48 & 1.93 \\
\hline
\end{tabular}

connect the panel to the L-frame and contacts take place between both parts.

Figure 6 shows strain time histories for the gauge located at location $S_{2}$ of the three test cases. Strains are given in $x$ - and $y$-direction at the same location. Gauge location $S_{2}$, which is located at $325 \mathrm{~mm}$ from the trailing edge on the center line, was selected to facilitate the comparison and assessment of the numerical methods - the main objective of the present work - and to avoid spurious effects induced by structural model boundaries, e.g. the contacts between the panel and the L-frame. The strain time histories at gauge $S_{2}$ agree well. Minor differences are found in terms of oscillation amplitudes in case 2122, which are attributed to the different natural time steps used in the simulations. Yet, mean strain values in the phase of constant pressure show differences of $11.3 \% / 0.7 \%$ (case $2122, x / y$ ) and $1.2 \% / 4.0 \%$ (case 3122 , $x / y$ ) from their common mean strain values.

These discrepancies are attributed to the code-specific differences in the computation of shell elements, link elements, contacts, and plasticity variables, which are inevitable. Based on a further simplification using a fully clamped panel, where the only differences of the models are the exact shell element formulation and the computation of plasticity variables, it was demonstrated that differences from their common mean strain values already occur. At the gauge location $S_{2}$ differences are $0.93 \% / 0.16 \%$ (case $2122, x / y$ ) and $3.1 \% / 2.9 \%$ (case $3122, x / y$ ).

\section{Simulation Results}

Having validated the structural models with reported differences, ALEFE and SPH-FE simulations for the three selected test cases of the benchmark experiment were conducted. In this section, the simulation results are compared to experimental data from the guided ditching experimental campaign (cf. Sec. 3.1). 




Figure 5: Comparison of upper surface strain distributions $\left(\varepsilon_{x x}\right)$ for simulations with ALE-FE software (top) and SPH-FE software (bottom) at $t=20 \mathrm{~ms}$. Contours are given in $[\mathrm{mm} / \mathrm{mm}]$. Structures refer to test cases 2122 (left) and 3122 (right). Half of the panel is shown to help the comparison.

\subsection{Pressure}

Being the source of the structural loading, pressure time histories are evaluated first. Figure 7 provides results for pressure probes $P_{4}$ and $P_{12}$ located on the center line $(y=0 \mathrm{~mm})$ at $x=125 \mathrm{~mm}$ and $x=400 \mathrm{~mm}$, respectively, from the trailing edge of the panels. These results are representative for the effects observed at all 18 pressure probe locations. Experimental pressure results were recorded only in test case 1122. Common for both numerical methods, pressure results uncover the strengths as well as the limitations of the fluid models. The employed methods both capture the delaying effect of the structural deformations on the pressure results. This delay grows while the panel immerses into the water, which shows as a growing difference in timing comparing pressures at probes $P_{4}$ and $P_{12}$. The ALE solution shows an excellent timing compared to the experimental data of case 1122 . In the other cases, the pressures in the ALE solution advance slower compared to the SPH solution. With the latter advancing faster in time, a growing time discrepancy arises as the specimens immerse into the fluid. This behavior is attributed to the lack of air and related phenomena such as air cushioning and ventilation, which are both expected to lower the acting hydrodynamic loads [1, 39] and thus delay the impact.

In general, the use of weakly-compressible schemes, i.e. employing an equation of state, results in numerical pressure oscillations that arise due to the "stiffness" of the EOS, which already for small density variations yields large pressure variations. The oscillations are generally stronger in the SPH 

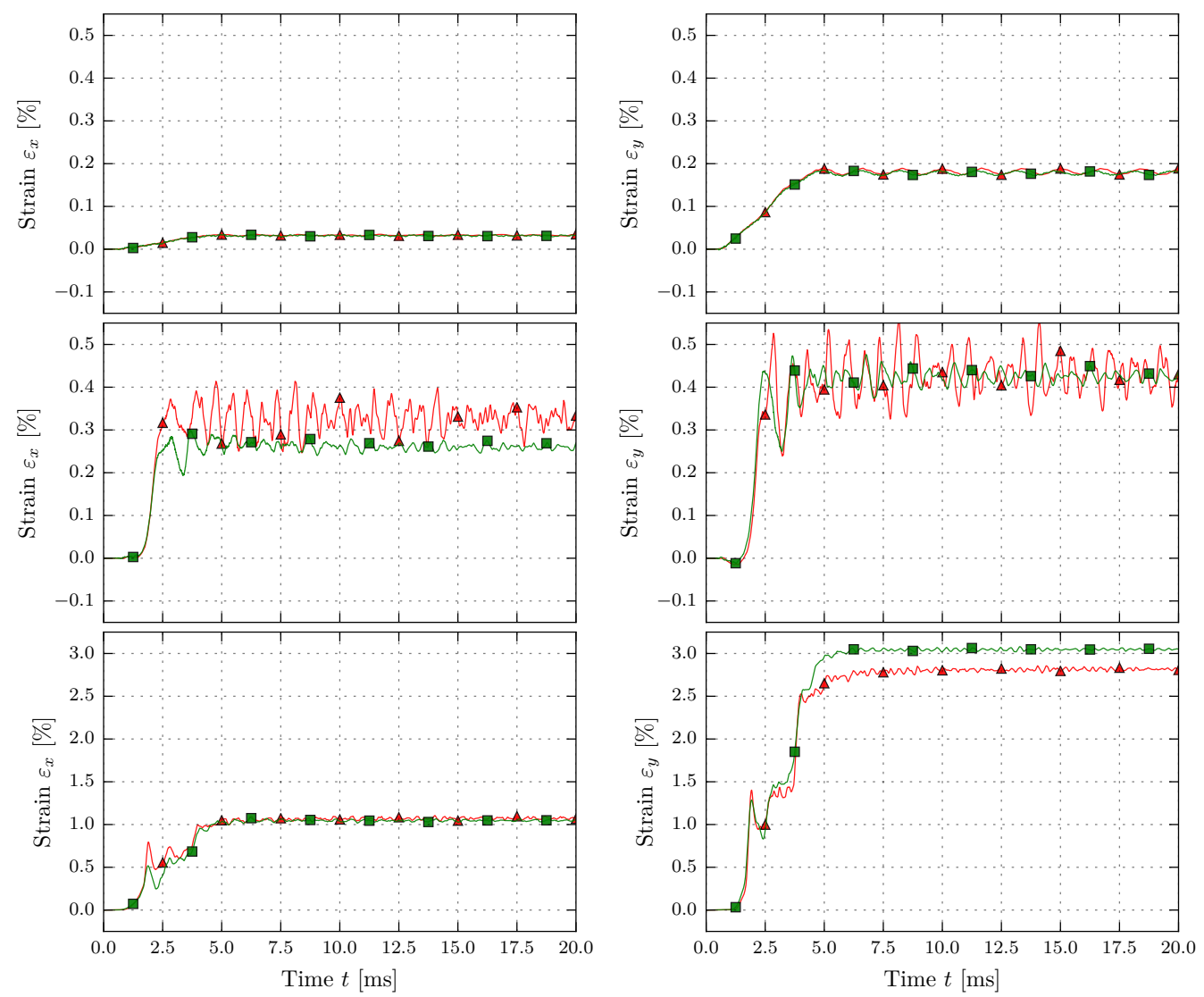

Figure 6: Comparison of strain time histories at gauge location $S_{2}$ for simulations with ALE-FE software (green solid lines and square markers) and SPH-FE software (red solid lines and triangle markers). Structures refer to test cases in the order 1122, 2122, and 3122 from top to bottom. 
solution than in the ALE solution, which is attributed to two main aspects. On the one hand, the particle volume is significantly larger compared to the cell volume, i.e. $720 \mathrm{~mm}^{3}$ for SPH versus $125 \mathrm{~mm}^{3}$ for ALE. On the other hand, the Lagrangian nature of SPH leads to an increasingly irregular particle distribution, which adversely affects the accuracy of the SPH algorithm. Just after the decay of the peak pressure, the pressure in the ALE simulations shows strong oscillations, which however quickly decay. In addition, the pressure measurement technique is affecting the oscillations recorded by the numerical pressure gauges: whereas in the ALE simulations, the gauges evaluate the pressure from one ALE cell, in the SPH simulations passive SPH particles (gauge particles) probe the pressure over a number of neighbor particles located inside their compact support domain [40], which leads to a variation in the number of particles used for the evaluation.

While the major shape of the pressure response is captured by both simulation methods, there are distinct differences in the ability to capture the highly transient pressure peak with its sharp rise and quick decay as well as the post-peak pressure level. In the ALE method, the contact stiffness affects the pressure peak values: a higher stiffness yields larger pressure peak values. ALE results show the characteristic pressure peak as observed in the experiments. In contrast, the measured SPH pressure results do not portray such sharp pressure peaks and also the measured pressure values are lower compared to ALE predictions and experimental data. This is assumed to be related to the employed pressure measuring technique in the SPH simulations. On the one hand, the size of the compact support of the gauge particles (i.e. the sampling domain) and, in addition, the present particle resolution are both considerably larger than the sensible element of the pressure probes used in the experiment. This leads to a smoothing effect and a lower measured pressure, while the local pressure acting on the structure may reach higher values. On the other hand, the gauge particles probe the pressure in a spherical region, which therefore also includes particles farther away from the surface of the structure, where due to the pressure gradient in normal direction to the structure the local pressure is lower. The latter could be circumvented by using a more complex pressure measurement technique, as for instance the one presented by Oger et al. [41], that permits limiting the sampling domain also in normal direction to the structure's surface. Yet, this should not compromise the computational efficiency as it involves computations of surface normals. 



Figure 7: Comparison of pressure time histories of probes $P_{4}$ (left) and $P_{12}$ (right) for ALE-FE (green solid lines and square markers) and SPH-FE (red solid lines and triangle markers) simulations against experimental data (black solid lines without markers). Test cases refer to 1122,2122 , and 3122 from top to bottom. 


\subsection{Normal Force}

Normal force time histories are shown in Fig. 8. They provide a measure of the overall load resulting from the fluid-structure interaction. The force time histories generally are in good qualitative agreement with the experimental data. Both numerical methods predict the initial slope, its reduction after the flow field has been established, and the abrupt decay after the leading edge immersion. Common for both numerical methods, normal force oscillations are lower for test cases with deformable specimens as structural deformations act as a filter of the highly transient and oscillating pressure that is acting on the structure. Another observation related to the occurrence of structural deformations is the local force peak just before the leading edge immersion that is predicted by both simulation methods for the cases with deformable panels. As shown by Siemann [1], this force peak is caused by the FSI at the front of the panel, where the structural deformation results in a negative local pitch angle of the panel. The timing of normal force results follows the observations made for pressure results, i.e. the SPH solution advances faster in time, whereas the ALE solution shows an excellent timing compared to the experimental data. This is particularly visible at the instant when the leading edge immerses into the water causing the normal force to decay rapidly. Normal force magnitudes are lower in the ALE solutions, which is attributed to leakage problems that can appear when the fluid flow moves nearly tangential to the structure. This is particularly true when the FSI is computed based on a penalty method as done in this work. There, the normal force acting on the water may be underestimated thus leading to leakage.

\subsection{Strain}

Strain time histories are evaluated in order to assess the accuracy of the structural response. Figure 9 shows the responses at gauge location $S_{2}$. Common for all test cases, the strain rate is lower for the ALE solution, which results from the underestimated rate of the pressure loading, i.e. the pressure rises too smooth and thus the structural loading lags. Both solutions considerably underestimate the experimental strain magnitude in test case 1122, which is attributed to the effects of model simplifications that will be discussed in Sec. 5.3. In test case 2122, both solutions capture the strain response with good agreement to the experimental data. Strain magnitudes at gauge location $S_{2}$ reach the upper elastic region. Yet, there is a time lead of the SPH solution and, in contrast, a time lag of the ALE solution. Large 


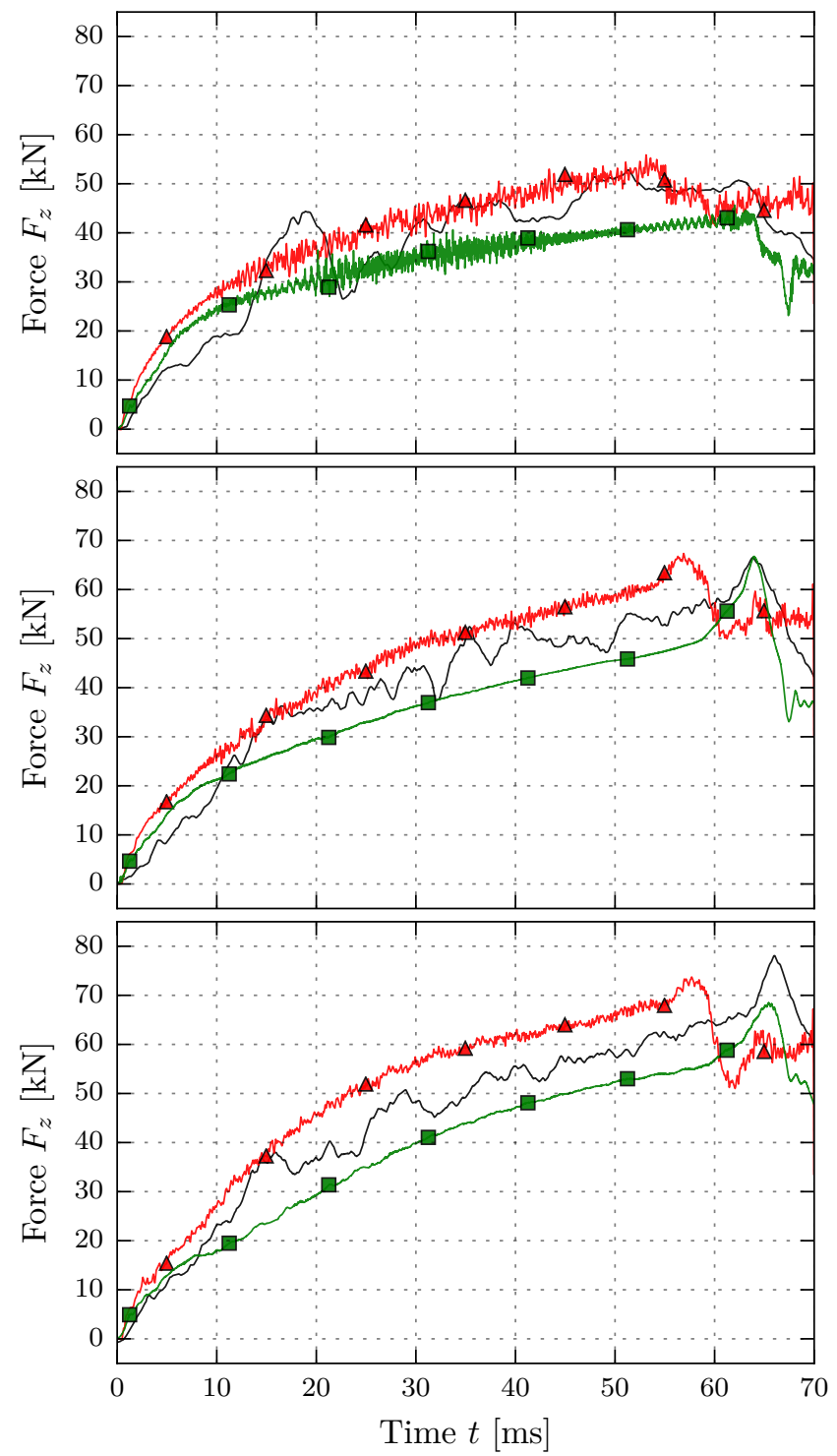

Figure 8: Comparison of normal force time histories for ALE-FE (green solid lines and square markers) and SPH-FE (red solid lines and triangle markers) simulations against experimental data (black solid lines without markers). Test cases refer to 1122, 2122, and 3122 from top to bottom. 



Figure 9: Comparison of strain time histories at gauge location $S_{2}$ for ALE-FE (green solid lines and square markers) and SPH-FE (red solid lines and triangle markers) simulations against experimental data (black solid lines without markers). Test cases refer to 1122, 2122 , and 3122 from top to bottom.

discrepancies are observed in test case 3122, where strains reach the plastic region. Both simulation results show a high strain rate in $x$-direction at the instant when the jet root passes the gauge location, whereas the experimental data features a smooth and steady rise of the strain. This observation is ascribed to the lack of capturing the detailed jet root shape in the simulations, which would require a further refinement of the fluid domain. Hence, in the simulations the pressure is applied more rapidly compared to the experiments, where the jet flowing along the structure already causes a loading before the arrival of the high-pressure zone located at the jet root. 


\section{Discussion}

\subsection{Effects of Plasticity Computation Method}

Validation simulations indicated a noticeable effect of the algorithm used to compute the plastic variables on the strain results. Due to its availability in both software packages, a Newton iterative algorithm was adopted for the comparative studies. Nevertheless, effects of the plasticity computation method on the simulation results were investigated based on additional ALEFE simulations using the radial return algorithm. Strain results recorded at the gauge locations did not show noticeable effects as they remained within the elastic range. Contour plots, however, revealed larger plastic strains, which were affected by the algorithm used to compute the plastic variables, occurring concentrated at the interface of the specimen and the L-frame. Hence, this change in the structural response did not lead to significant differences in the simulation results. Nevertheless, the use of the radial return algorithm should be favored as it is more accurate compared to the Newton iterative algorithm [42].

\subsection{Effects of Air}

In order to investigate the effects of air on the simulation results, an ALE-FE simulation of case 1122 with a modified air material was conducted. Therein, the air density was decreased by a factor of one thousand to an artificial value of $1.2 \cdot 10^{-12} \mathrm{~kg} / \mathrm{mm}^{3}$. Although the simulation became unstable towards the end of the computation, several observations could be established. Pressure time histories were very similar to the ones in the simulation with the initial air density. They generally showed no effect on the timing, yet lower peak values followed by a phase with significantly lower and even negative relative pressures caused by local instabilities due to a decrease of the density to very small values near zero that were unphysical. This later led to the premature termination of the computation caused by a negative density in one ALE cell. Due to the changes of the pressure acting on the structure, the normal force was lower during the first $25 \mathrm{~ms}$ of the impact, whereas afterward the normal force time histories were quasi-identical until the termination that came along with growing oscillations of the normal force. Consequently, the lower normal force yield very similar strain time histories yet with reduced peak values. In summary, the pursued way to investigate effects of air did not permit observing the anticipated results due to 
air cushioning, such as lower peak pressures, lower normal force, and smaller strain magnitudes.

\subsection{Effects of Model Simplifications}

The absence of the guide structure in the model, which was discarded to avoid additional differences in the structural model due to the use of two software packages, resulted in additional discrepancies with respect to the experimental results. In order to quantify these discrepancies, SPH-FE simulation using a structural model including the guide structure and an elastic trolley model were conducted. Figure 10 compares numerical results for the two models, i.e. with and without the guide structure, against experimental results for all three test cases. The discrepancies of the normal force magnitudes were in the order of $8 \%$ to $12 \%$ for the regarded test cases. Normal forces were generally larger when there was no guide structure because the evasive motion observed in the experiment was suppressed and thus the acting vertical velocity remained larger. Furthermore, the timing of the numerical results was slightly worse without using the guide model. This can be explained by the absence of the reduction of the vertical velocity, which delayed the immersion in the cases with the guide model. Despite the reduced correlation due to the absence of the guide structure, the superimposed oscillations were lower for all simplified models, which is attributed to the use of the rigid body trolley model. Strain time histories are presented in Fig. 11 for gauge $S_{2}$. For case 1122 strain magnitudes were highly affected by the model simplification. In particular, the rigid trolley model was responsible for the differences of the structural response near the interface of the specimen and the L-frame. In contrast, no significant differences occurred in case 2122. Finally, case 3122 showed minor differences, yet the strain magnitudes were smaller in the simulation with the model simplification. Overall, strain results indicated the importance of the structural modeling that affected the structural response depending on the stiffness of the specimen.

In addition, the effects due to the use of a simplified, rigid trolley model were investigated. Therefore, further ALE-FE simulations with an elastic trolley model were conducted. Results showed no effect on the normal force. In comparison with above findings, the observed sensitivity of the normal force on the model simplifications can therefore be ascribed to the presence of the guide structure. However, there was an important effect on the strain time histories in case 1122 as shown in Fig. 12. Similar to the observations 


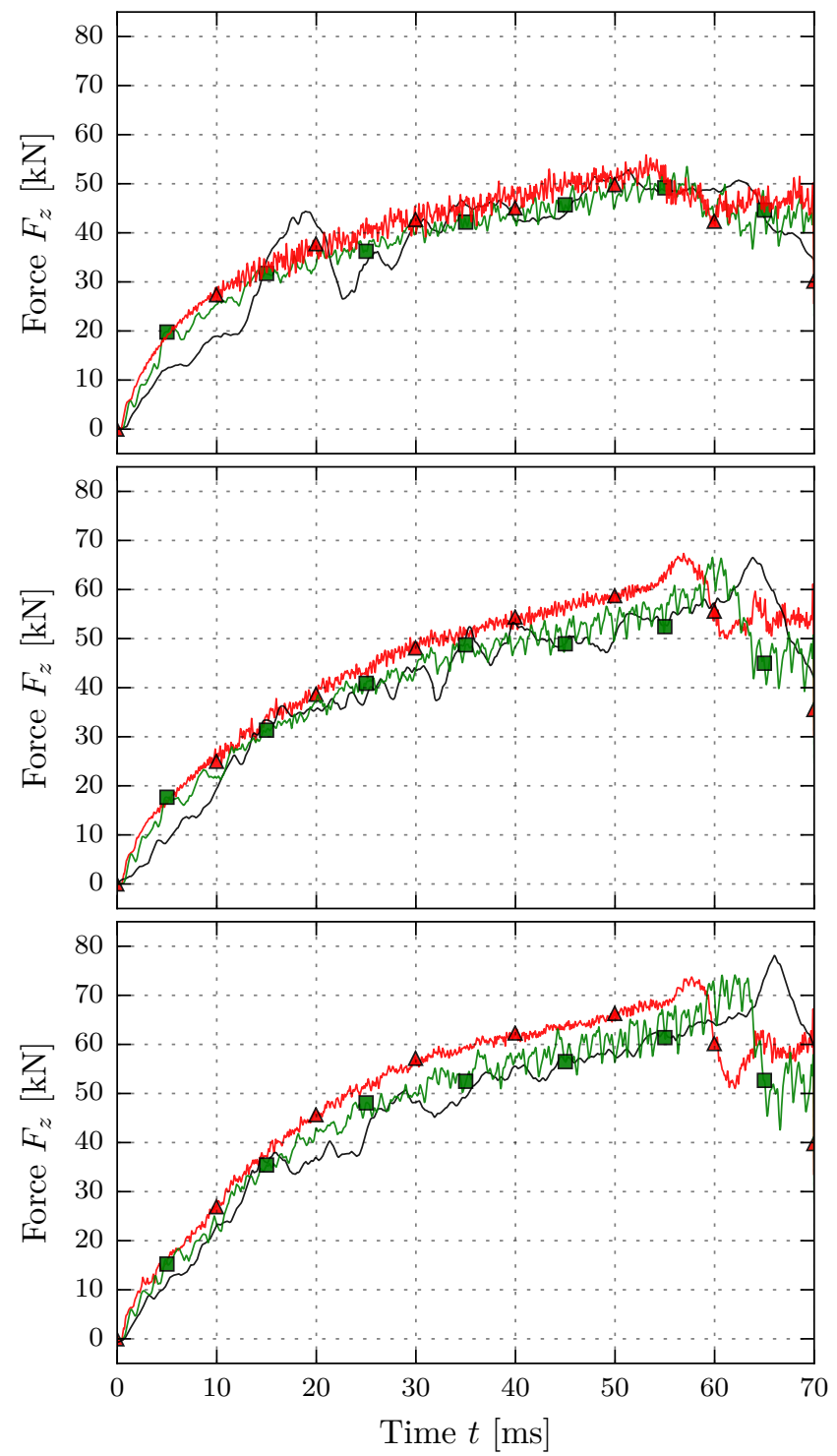

Figure 10: Comparison of normal force time histories for SPH-FE simulations without (green solid lines and square markers) and with (red solid lines and triangle markers) model simplification against experimental data (black solid lines without markers). Test cases refer to 1122,2122 , and 3122 from top to bottom. 



Figure 11: Comparison of strain time histories at gauge location $S_{2}$ for SPH-FE simulations without (green solid lines and square markers) and with (red solid lines and triangle markers) model simplification against experimental data (black solid lines without markers). Test cases refer to 1122,2122 , and 3122 from top to bottom. 



Figure 12: Comparison of strain time histories at gauge location $S_{2}$ for ALE-FE simulations with rigid (green solid lines and square markers) and elastic (blue solid lines and triangle markers) trolley model against experimental data (black solid lines without markers). Test case 1122 .

in Fig. 11, strain magnitudes were found to be sensitive to the stiffness of the trolley model. Future studies could further address the sensitivity of the structural response to the discretization of the trolley and in particular the L-frame as well as the joint modeling.

\subsection{Further Aspects}

The resolution of the structural model was identical for both computational methods. It defines the resolution of the ALE fluid domain, which must be smaller than the structural resolution to warrant proper functionality of the coupling; however, the resolution of the SPH fluid domain could not be refined as much because of constrains regarding the related computational effort. Consequently, a comparison of the computational effort between the two computational methods cannot be established. Furthermore, the pressure measurement in each method is affected by the difference in fluid domain resolution as well as the measurement technique. These points could be addressed in further parametric studies regarding a similar yet two-dimensional water impact test case and evaluating local as well as global results.

The present study also indicated coupling effects between fluid and structure in the ALE simulations. When the fluid material motion is tangential to the structure, leakage may occur because contact forces can be underestimated. Additionally, the contact height was found to affect the rate of the pressure acting on the structure. The latter could be further investigated based on above-mentioned parametric studies concerned with the fluid domain resolution or by using Lagrange multipliers for the fluid-structure coupling. 
This study was limited to one impact condition, i.e. the combination of impact velocities and pitch angle, with limited structural deformation and no failure. For impact conditions leading to higher loads, however, findings may vary as the structural failure in terms of material or joint failure could develop. Hence, future studies could compare the computational methods in terms of their capabilities and limitations when structural failure occurs.

\section{Conclusion}

This work investigated the capabilities and limitations of two coupled fluid-structure computational methods, namely ALE-FE and SPH-FE approaches. The use of different software packages for simulations based on each method impeded the present study, but the comparison was concentrated as far as possible on the fluid methods. Therefore, the similarity of the structural models in each software package was extensively studied and finally demonstrated with the reported accuracy. Results of benchmark simulations of guided ditching experiments demonstrated that both approaches were capable of simulating the fluid as well as the structural response of quasi rigid as well as deformable specimen impacting water under conditions representative of aircraft ditching. The ALE-FE and the SPH-FE approaches were able to handle large fluid displacements in conjunction with rapidly moving structures undergoing significant structural deformations.

Despite continuous advances in computing technologies, e.g. high performance computing, advanced modeling strategies, etc., the main limitation for both approaches is their large run time. In order to apply these approaches to large realistic aircraft structural models, a further speed-up is required.

\section{Acknowledgement}

This work was performed as part of the common research project ADAWI ${ }^{6}$, which was supported by Onera and DLR.

\section{References}

[1] M. H. Siemann, Numerical and Experimental Investigation of the Structural Behavior during Aircraft Emergency Landing on Water, Doctoral thesis, University of Stuttgart, 2016.

\footnotetext{
${ }^{6}$ Assessment of Ditching and Water Impact, 2015-2017
} 
[2] H. Climent, L. Benítez, F. Rosich, F. Rueda, N. Pentecôte, Aircraft Ditching Numerical Simulation, in: 25th International Congress of the Aeronautical Sciences, Hamburg, Germany, 2006.

[3] N. Toso, Contribution to the Modelling and Simulation of Aircraft Structures Impacting on Water, Doctoral thesis, University of Stuttgart, doi: 10.18419/opus-3823, 2009.

[4] L. Benítez Montañés, H. Climent Máñez, M. Siemann, D. Kohlgrueber, Ditching Numerical Simulations: Recent Steps in Industrial Applications, in: Aerospace Structural Impact Dynamics International Conference, Wichita, USA, 2012.

[5] R. Ortiz, J. L. Charles, J. F. Sobry, Structural Loading of a Complete Aircraft Under Realistic Crash Conditions: Generation of a Load Database for Passenger Safety and Innovative Design, in: 24th International Congress of the Aeronautical Sciences, 2004.

[6] K. Hughes, R. Vignjevic, J. Campbell, Experimental observations of an $8 \mathrm{~m} / \mathrm{s}$ drop test of a metallic helicopter underfloor structure onto water: part 2, Journal of Aerospace Engineering 221 (2007) 679-690, ISSN 0954-4100, doi:10.1243/09544100JAERO228.

[7] K. Hughes, J. Campbell, R. Vignjevic, Application of the finite element method to predict the crashworthy response of a metallic helicopter under floor structure onto water, International Journal of Impact Engineering 35 (5) (2008) 347-362, doi:10.1016/j.ijimpeng.2007.03.009.

[8] K. Hughes, J. Campbell, Helicopter crashworthiness: a chronological review of water impact related research from 1982 to 2006, Journal of American Helicopter Society 53 (4) (2008) 429-442, doi: 10.4050/JAHS.53.429.

[9] R. N. Bingman, Water Impact Loads on Structures, M.sc. thesis, Air University, 1967.

[10] S.-L. Chuang, Investigation of Impact of Rigid and Elastic Bodies with Water, Tech. Rep., Department of the Navy Naval Ship Research and Development Center, Washington, USA, 1970. 
[11] R. Panciroli, S. Abrate, G. Minak, A. Zucchelli, Hydroelasticity in water-entry problems: Comparison between experimental and SPH results, Composite Structures 94 (2012) 532-539, ISSN 02638223, doi: 10.1016/j.compstruct.2011.08.016.

[12] T. D. Allen, Mechanics of Flexible Composite Hull Panels Subjected to Water Impacts, Ph.D. thesis, University of Auckland, 2013.

[13] J. Donea, S. Giuliani, J. P. Halleux, An Arbitrary Lagrangian-Eulerian Finite Element Method for Transient Dynamic Fluid-Structure Interactions, Computer Methods in Applied Mechanics and Engineering 33 (1982) 689-723, doi:10.1016/0045-7825(82)90128-1.

[14] J. Donea, A. Huerta, Finite Element Methods for Flow Problems, John Wiley \& Sons, doi:10.1002/0470013826, 2003.

[15] M. Souli, D. J. Benson, Arbitrary Lagrangian Eulerian and FluidStructure Interaction: Numerical Simulation, Wiley-ISTE, doi: 10.1002/9781118557884, 2010.

[16] R. A. Gingold, J. J. Monaghan, Smoothed particle hydrodynamics - Theory and application to non-spherical stars, Monthly Notices of the Royal Astronomical Society 181 (November) (1977) 375-389, doi: 10.1093/mnras/181.3.375.

[17] T. Belytschko, Y. Y. Lu, L. Gu, Element-Free Galerkin Methods, International Journal for Numererical Methods in Engineering 37 (1994) 229-256, ISSN 00295981, doi:10.1002/nme.1620370205.

[18] S. Beissel, T. Belytschko, Nodal integration of the element-free Galerkin method, Computer Methods in Applied Mechanics and Engineering 139 (1) (1996) 49-74, doi:10.1016/S0045-7825(96)01079-1.

[19] S. Abrate, Hull Slamming, Applied Mechanics Reviews 64 (6), doi: 10.1115/1.4023571.

[20] M. Anghileri, L.-M. L. Castelletti, E. Francesconi, A. Milanese, M. Pittofrati, Survey of numerical approaches to analyse the behavior of a composite skin panel during a water impact, International Journal of Impact Engineering 63 (2014) 43-51, ISSN 0734743X, doi: 10.1016/j.ijimpeng.2013.08.008. 
[21] K. Hughes, R. Vignjevic, J. Campbell, T. DeVuyst, N. Djordjevic, L. Papagiannis, From Aerospace to Offshore: Bridging the Numerical Simulation Gaps-Simulation advancements for fluid structure interaction problems, International Journal of Impact Engineering 61 (2013) 48-63, doi:10.1016/j.ijimpeng.2013.05.001.

[22] B. Guo, P. Liu, Q. Qu, J. Wang, Effect of pitch angle on initial stage of a transport airplane ditching, Chinese Journal of Aeronautics 26 (1) (2013) 17-26, ISSN 10009361, doi:10.1016/j.cja.2012.12.024.

[23] Q. Qu, M. Hu, H. Guo, P. Liu, R. Agarwal, Study of ditching characteristics of transport aircraft by global moving mesh method, Journal of Aircraft 52 (5) (2015) 1550-1558, ISSN 00218669, doi:10.2514/1.C032993.

[24] E. E. McBride, L. J. Fisher, Experimental investigation of the effect of rear-fuselage shape on ditching behavior, TN 2929, Tech. Rep., NACA, Langley Field, USA, 1953.

[25] N. Pentecôte, D. Kohlgrüber, Full-scale Simulation of Aircraft Impacting on Water, in: International Crashworthiness Conference, San Francisco, USA, 2004.

[26] P. H. L. Groenenboom, M. H. Siemann, Fluid-structure interaction by the mixed SPH-FE method with application to aircraft ditching, The International Journal of Multiphysics 9 (3) (2015) 249-265, doi: 10.1260/1750-9548.9.3.249.

[27] J. Donea, A. Huerta, J.-P. Ponthot, A. Rodríguez-Ferran, Arbitrary Lagrangian-Eulerian Methods, in: E. Stein, R. de Borst, T. J. Hughes (Eds.), Encyclopedia of Computational Mechanics, chap. 14, JohnWiley \& Sons, 1 edn., ISBN 0-470-84699-2, 2004.

[28] O. M. Faltinsen, Hydrodynamics of High-Speed Marine Vehicles, Cambridge University Press, ISBN 9780521845687, doi: 10.1017/CBO9780511546068, 2005.

[29] J. J. Monaghan, Particle methods for hydrodynamics, Computer Physics Reports 3 (1985) 71-124, ISSN 01677977, doi:10.1016/01677977(85)90010-3. 
[30] J. J. Monaghan, Simulating Free Surface Flows with SPH, Journal of Computational Physics 110 (1994) 399-406, doi:10.1006/jcph.1994.1034.

[31] F. Casadei, J. P. Halleux, An algorithm for permanent fluid-structure interaction in explicit transient dynamics, Computer Methods in Applied Mechanics and Engineering 128 (3-4) (1995) 231-289, ISSN 00457825, doi:10.1016/0045-7825(95)00843-8.

[32] F. Casadei, S. Potapov, Permanent fluid-structure interaction with nonconforming interfaces in fast transient dynamics, Computer Methods in Applied Mechanics and Engineering 193 (39-41 SPEC. ISS.) (2004) 4157-4194, ISSN 00457825, doi:10.1016/j.cma.2003.06.002.

[33] F. Casadei, N. Leconte, Coupling finite elements and finite volumes by Lagrange multipliers for explicit dynamic fluid-structure interaction, International Journal for Numerical Methods in Engineering 86 (2011) 1-17, doi:10.1002/nme.3042.

[34] D. Delsart, B. Langrand, A. Vagnot, Evaluation of an Euler/Lagrange coupling method for the ditching simulation of helicopter structures, in: 5th International Conference on Fluid Structure Interaction, Royal Mare Village, Crete, Greece, 259-268, doi:10.2495/fsi090241, 2009.

[35] A. Iafrati, Experimental investigation of the water entry of a rectangular plate at high horizontal velocity, Journal of Fluid Mechanics (2016) 637672ISSN 0022-1120, doi:10.1017/jfm.2016.374.

[36] A. Iafrati, S. Grizzi, M. H. Siemann, L. Benítez Montañés, High-speed ditching of a flat plate: Experimental data and uncertainty assessment, Journal of Fluids and Structures 55 (May) (2015) 501-525, ISSN 10958622, doi:10.1016/j.jfluidstructs.2015.03.019.

[37] D. Kohlgrüber, A. Vigliotti, V. Weissberg, H. Bartosch, Numerical simulation of a composite helicopter sub-floor structure subjected to water impact, in: American Helicopter Society 60th Annual Forum, Baltimore, USA, 2004.

[38] H. Huth, Influence of Fastener Flexibility on the Prediction of Load Transfer and Fatigue Life for Multiple-Row Joints, in: Fatigue in Mechanically Fastened Composite and Metallic Joints, 221-250, 1985. 
[39] Z. H. Ma, D. M. Causon, L. Qian, C. G. Mingham, T. Mai, D. Greaves, A. Raby, Pure and aerated water entry of a flat plate, Physics of Fluids 28 (1), ISSN 10897666, doi:10.1063/1.4940043.

[40] M. Siemann, P. Groenenboom, Pressure Evaluation at Arbitrary Locations in SPH Water Impact Simulations, in: PARTICLES 2013, Stuttgart, Germany, 961-973, 2013.

[41] G. Oger, M. Doring, B. Alessandrini, P. Ferrant, Two-dimensional SPH simulations of wedge water entries, Journal of Computational Physics 213 (2) (2006) 803-822, ISSN 00219991, doi:10.1016/j.jcp.2005.09.004.

[42] J. C. Simo, T. J. R. Hughes, Computational Inelasticity, Springer, ISBN 978-0-387-97520-7, doi:10.1007/b98904, 1998. 